\title{
Effects of Iron Oxyhydroxide Nanoparticle Aggregation via Freezing and Drying on Zn(II) Adsorption and Retention
}

\footnotetext{
RYLY YEE ${ }^{1} \&$ CHRISTOPHER S. KIM ${ }^{2}$

1'yee@chapman.edu

${ }^{2}$ cskim@chapman.edu

Iron (oxy)hydroxides are commonly found in the nearsurface environment and play a significant role in metal fate and transport in contaminated systems. Iron oxyhydroxides often initially precipitate as nanosized particles but readily aggregate in natural aquatic systems, forming an important component in soils, sediments, and mine drainage outflows. Iron oxyhydroxides are also highly effective at adsorbing and retaining dissolved metals, which can be further incorporated into aggregate structures as structural impurities and lead to the formation of localized nano- or micro-precipitates.

We investigated the effects of iron oxyhydroxide nanoparticle aggregation via suspension freezing and drying on $\mathrm{Zn}^{2+}$ metal ion adsorption and retention. Iron oxyhydroxide nanoparticles were synthesized through a flash microwave nucleation process followed by dialysis, resulting in monodisperse suspensions of $\sim 5$-nm diameter nanoparticles. Separate suspensions were then frozen at $-80^{\circ} \mathrm{C}$ for $0-65$ minutes or dried under forced airflow at room temperature. The resulting aggregates were analyzed with dynamic light scattering (DLS) to determine the average aggregate size followed by $\mathrm{Zn}^{2+}$ macroscopic adsorption/desorption experiments to characterize $\mathrm{Zn}$ uptake/retention behavior to the nanoparticle aggregates.

Both aggregation pathways indicate that during the last $20 \%$ of liquid water frozen/evaporated, the average aggregate hydrodynamic radius increases exponentially, with similar trends in growth behavior for freezing/drying. The adsorption/desorption experiments show that initial $\mathrm{Zn}$ adsorption declines exponentially with particle size, while the fraction of retained $\mathrm{Zn}$ after desorption is induced by lowering suspension $\mathrm{pH}$ is relatively constant across multiple aggregate sizes. Studying the correlation between the freezing/drying extent and the aggregate size allows for the prediction of aggregate conditions that can maximize metal ion uptake and retention.
} 